\title{
Urgences
}

\section{Duchampienneries : derniers détails, vite}

\section{André Gervais}

Numéro 20, mai 1988

Appellation contrôlée

URI : https://id.erudit.org/iderudit/025488ar

DOI : https://doi.org/10.7202/025488ar

Aller au sommaire du numéro

Éditeur(s)

Urgences

ISSN

0226-9554 (imprimé)

1927-3924 (numérique)

Découvrir la revue

Citer ce compte rendu

Gervais, A. (1988). Compte rendu de [Duchampienneries : derniers détails, vite]. Urgences, (20), 78-80. https://doi.org/10.7202/025488ar d'utilisation que vous pouvez consulter en ligne.

https://apropos.erudit.org/fr/usagers/politique-dutilisation/ 


\section{Duchampienneries: der- niers détails, vite}

Il y a six mois - en octobre 1987 -, c'était la double sfêtex à Marcel Duchamp, artiste et anartiste français et américain. A la Nova Scotia College of Art and Design (Halifax), un colloque et au musée de Philadelphie, un symposium signalaient, en anglais mais aussi en français, qu'il y a cent ans, en 1887 , naissait dans un petit village quasi flaubertien, un petit village de Normandie donc, celui qui deviendra, entre autres pseudonymes, Rrose Sélavy. Je ne dirai rien ici de ces manifestations importantes et particulièrement diversifiées (je pense à tout ce qui était, à Philadelphie, ooff museum*) auxquelles j'ai assisté.

Plutôt, quelques mots et indications sur les dernières publications (pas toutes, un choix seulement) dont j'aurai eu vent durant ces six derniers mois.

À Halifax, début octobre, on apprend qu'est paru, en juillet probablement, hors-les-murs d'Yuetot (Normandie), le dernier numéro de Rrosopopées, petite revue confidentielle dirigée et rédigée par Jacques Caumont et Jennifer Gough-Cooper (ceux qui ont fait le Plan pour écrire une vie de Marcel Duchamp, le premier des quatre volumes du catalogue de l'exposition $\mathrm{MD}$ à Beaubourg, en 1977). Ce numéro, intitulé EpheMeriDes on and about Marcel Duchamp, contient un choix de 87 aévénements» tirés de la correspondance et d'autres sources: du 25 juillet au 7 août, de 1887 (naissance: 28 juillet) à 1966. Certains points intéressants: quelques-uns des petits cadeaux donnés par MD à ses amis ou reçus d'eux, quelques signatures particulières et des détails sur une oeuvre jusqu'ici inconnue (des délices de Kermoune, 1958). Ce numéro est accompagné, pour les abonnés, d'un supplément itlustré contenant des renseignements inédits sur d'autres détails: sur Simone Simon, jeune actrice peu connue du cinéma français, qui aurait servi de modèle au mannequin habillé par $\mathrm{MD}$ en Rrose Sélavy à l'Exposition internationale du Surréalisme (Paris, 1938), par exemple. On voit déjà comment le nom masculin (comme, inversement, le prénom Marcel) et le prénom féminin (comme, inversement, le nom Sélavy) de cette actrice a pu jouer dans le choix.

À Philadelphie, vers la fin octobre, on apprend que vient enfin d'être publié par le musée le manuel d'instructions d'Etant donnés: $1^{\circ}$ la chute d'eau $2^{\circ}$ le gaz d'éclairage, oeuvre faite à $\mathrm{New}$ York en secret de tous les critiques et de tous les amis (sauf de Teeny, sa fernme) pendant vingt ans (1946-1966) et dévoilée seulement en 1969 , neuf mois après la mort du principal désintéressé. $\mathrm{Ce}$ cahier de montage et démontage, rédigé en 1966 ou 1967 et illustré de photos prises depuis le début des années 60 , selon toute vraisemblance, est reproduit photographiquement et en couleurs, le tout littéralement, si je puis dire: le format, le page-par-page, les rabats, tout est scrupuleusement reproduit. Un document unique, donc, et en ce sens tout à fait dans le sillon tracé par la Boîte Verte, recueil de notes et de dessins publié en 1934 par MD. Certains détails, qui avaient été dévoilés par Jean-François Lyotard et André Gervais dans leurs livres respectifs (1977 et 1984), sont maintenant pourvus de leur contexte spécifique. S'il manque les photographies de deux factures (dont celle du magasin de Carlstadt, New Jersey, où il se procure en 1963 le moteur de la chute d'eau) qui étaient dans un 
exemplaire disponible seulement privément et que j'ai pu consulter au musée en 1978, il est ajouté la troisiéme de couverture de ce cahier qui n'est autre qu'un ordinaire cahier à anneaux: xed DORET $v *$ et $*$ c'est une création DORET * peut-on y lire, entre autres. Je n'hésiterai pas à reconnaître là, motivant le choix, telles indications métatextuelles: d'une part les initiales des deux premiers mots du titre, d'autre part cette femme couchée sur le dos et présentant au regardeur sa raie, ce qui est bien ce que l'on voit, entre autres, comme chacun sait.

On constate également, à la librairie du musée, qu'une jeune revue newyorkaise intéressée à la littérature et à l'architecture, Sites, vient de publier dans son numéro 19 une trentaine de pages relatives à Etant donnés... Un article de Paul Matisse, gendre de MD et technicien bénévole lors du démontage (dans le studio newyorkais) et du remontage (au musée de Philadelphie), puis, surtout, une entrevue avec Denise Browne Hare, amie de longue date des Duchamp et photographe: plusieurs photographies de studio et de l'oeuvre, prises à la fin 1968, tout de suite après la mort de MD, sont ici publiées (republiées, en fait, étant déjà parues dans le catalogue de l'expostion MD à Barcelone en 1984) et commentées. L'intérêt de ces photographies - et de celles accompagnant le manuel d'instructions est de proposer de l'oeuvre et de son environnement (ou cursus d'envies) un ensemble de points de vue en quelque sorte sacrilèges (et qui, pour cette raison sans doute, n'auront vraiment été présentables que bien plus tard): n'y voiton pas, comme rarement cela est mis à notre disposition, tant le laboratoire que la solution (de continuité)?

Dans la foulée du revampage, si je puis dire, de ses salles MD, ouvertes depuis 1954 (exhibits augmentés, petits cartons refaits, etc.), le musée de Philadelphie republie, avec une illustration complétée (entre autres, d'une Etude inédite et datée circa 1947), les Reflec- tions on a New Work by Marcel Duchamp par Anne d'Harnoncourt et Walter Hopps, parues en 1969 (à l'occasion du vernissage d'Etant donnés...) et déjà augmentées d'une postface en 1973. C'était la première étude sur l'oeuvre et c'est encore l'une des plus détaillées (68 p.). Le musée de Philadelphie, mixte de Louvre et de Beaubourg (pour faire une rapide comparaison transatlantique), est le musée, faut-il le rappeler, qui contient le plus grand fonds d'oeuvres et d'archives duchampiennes. En ce sens, il invite particulièrement au D.tour.

Fin 1987, la revue Canadian Ant (Toronto) publie dans son numéro d'hiver 1987 un article de Dennis Reid intitulé *Marcel Duchamp in Canada*, en fait essentiellement à Toronto entre 1913 (où l'on parle brièvement dans un journal de la célébre toile $N u$ descendant un escalier qui fait alors un malheur à New York et à Chicago) et 1968 (où MD, Teeny et John Cage, entre autres. proposent un spectacle unissant échecs et musique, et justement intitulé Reunion). Et une interview inédite, faite à New York en 1965, par Don Bell. A quand un article sur MD et le reste du Canada, MD et le Québec particulièrement? Je verse déjà au dossier l'amitié, à la fin des années 50, de Gilles Hénault, poète, journaliste et critique d'art montréalais, et de Michel Sanouillet, alors professeur à l'Université de Toronto et éditeur des écrits de MD (parus au Terrain vague en 1959, reparus chez Flam. marion en 1975), et cette interview, faite à New York en 1960, par le critique d'art montréalais Guy Viau (et, bien que diffusée à la télévision de Radio-Canada la même année, inconnue des bibliographies duchampiennes).

Toujours fin 1987, paraissent à Munich, chez Verlag Silke Schreiber, les Lettres à Marcel Jean, en français et en traductions allemande et anglaise. Vingt lettres (1952-59, 1967-68). Les plus importantes sont les premières, écrites à une époque où il n'y a aucun livre ni aucun catalogue sur l'oeuvre, parce 
qu'elles nous livrent des renseignements de première main (à confronter avec les réponses écrites aux questions, envoyées quand?, de Robert Lebel et à celles, envoyées en 1960, de Serge Stauffer). Je ne citerai que deux brefs paragraphes du début de la première lettre, qui donne le ton et pose la problématique:

Il est seulement curieux de constater combien la mémoire est fragile même pour les époques importantes de la vie.

C'est d'ailleurs ce qui explique la fantaisie heureuse de l'histoire (sans point final)

Ceci, par exemple, permet-il d'expliquer toute l'importance que MD accorde à une chronologie loose de son oeuvre et de sa vie, en en distendant un peu effets et faits? Le critique (ou analyste de textes) et, plus simplement, le biographe n'ont-ils pas, nécessairement, un coin d'attaque autre: ne leur faut-il pas, d'emblée pour ainsi dire, un texte bien établi et des événements bien datés, par exemple? C'est la même chose, il va sans dire, pour l'historien d'art.

Début 1988, on me signale que Dora Vallier vient de publier un tout petit livre intitulé Art, anti-art et non-art (Caen, L'Echoppe, novembre 1986, 29 p.) dédié aA la mémoire de Marcel Duchamp», petit livre qui est la réédition, augmentée d'une postface, d'un article paru dans La NRF en 1969 et inconnu des bibliographies duchampiennes. L'intérêt de ce livre tient, entre autres ( $p$. 14-15 et 26), à l'importance d'un *aveu surgi à l'improviste vers la fin du long entretien* qu'elle avait eu avec MD, aveu à propos de sa lecture, probablement dès les années 10, de l'oeuvre de Nietzsche. A creuser, comme dit Mallarmé.

Enfin, dans ses numéros de février et de mars 1988 , le magazine House \& Garden (eh oui, après Life dès 1950 et 1952, puis Vogue en 1963) parle de MD. D'abord (p. 100-107 et 186), à l'occasion d'un reportage photographique sur la maison de campagne où habite Teeny Duchamp, prẻs de
Fontainebleau. Ensuite (p. 178-185 et 224), à l'occasion d'un reportage lui aussi photographique sur la nouvelle maison de Dennis Hopper (eh oui, l'acteur américain bien connu) à Venice, California. On y apprend qu'Hopper a côtoyé MD lors de sa rétrospective (organisée par Walter Hopps) à Pasadena en 1963 , et ce d'assez près pour qu'il signe l'enseigne de l'hôtel où il habitait. Ce ready-made, intitulé *simplement* (!) et autoréférentiellement Signed Sign, d'une part renvoie à la Boîte Verte (via le nom de l'hôtel: Hotel Green), d'autre part entre en résonance avec le titre des écrits (Duchamp du signe, surgi à Paris en 1965). Quand on sait que ce readymade est depuis ce jour dans la collection de l'acteur, qu'il n'est dans aucun catalogue et n'a jamais été publiquement exposé, et que le titre des écrits ne sera publié que dix ans plus tard (dans l'édition Flammarion), on voit assez facilement la difficulté (et l'enjeu) qu'il y a à travailler sur un corpus duchampien dûment constitué. Tout est utile - des publications de musée de Philadelphie aux articles glamour de House \& Garden - pour constituer le terreau d'une lecture toujours plus précise, toujours mieux documentée des abagatelles végétales» duchampiennes.

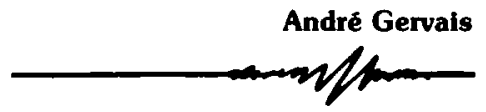

\section{Une littérature spécifique?}

Jean Cossette, Sayam! La bete du Lac Matapédia... illustré par Fernande Forest, Rimouski, Editeq, 1986.

Gilbert Dupuis, Les étoiles de la Neigette, illustré par Hélène Couture, Rimouski, Editeq, 1987.

Le texte de Jean Cossette est accompagné d'un disque 33 tours interprété par une équipe de comédiens. Il est introduit par une chanson inédite, composée et interprétée par Serge Arsenault et une chorale d'enfants. 